\title{
Cooling a Solid Disc with Uniform Heat Generation Using Inserts of High Thermal Conductivity within the Constructal Design Platform
}

\author{
F. Sharfi ${ }^{a}$, R. M. Salimpuor ${ }^{a}$, and A. Campo ${ }^{b, *}$ \\ ${ }^{a}$ Department of Mechanical Engineering, Ishafan University of Technology, Ishafan, Iran \\ ${ }^{b}$ Department of Mechanical Engineering, The University of Vermont, Burlington, VT 05405, USA
}

\begin{abstract}
In the present study, the problem of cooling a solid disc by way of placing inserts with high thermal conductivity was examined analytically and numerically within the platform of Constructal Theory. The work was accomplished using a fixed amount of a highly conductive material distributed in the form of incomplete inserts from the center (sink). Using Constructal Theory, the magnitudes of the heat resistances in the radial and the branching configurations were calculated analytically. Additionally, to validate the analytical solution, a numerical solution with the Finite Element Method was employed. The one-to-one comparison between the two distinct results reveals a good agreement. In the present case, the length of the inserts was different from the disc radius viz. a new degree of freedom was considered and the solution was remarkably different from the case involving a complete insert. The heat resistance was minimized with respect to the aspect ratio in order to determine the optimal number of inserts as well as the disc radius. It was demonstrated that within in a certain range of parameters, the heat conduction performance of incomplete inserts in the solid disc surpasses the heat conduction performance of standard complete inserts.
\end{abstract}

Keywords: Cooling, Solid Disc, Uniform Heat Generation, Incomplete Inserts, High Thermal Conductivity, Constructal Theory.

\section{Introduction}

In the highly demanding area of thermal design of electronic devices, primary attention should be paid at reducing the maximum thermal resistances along a specified thermal path and/or providing parallel paths for the heat removal from a critical volume or component (Bar-Cohen et al. [1]). As the length scales of the electronic devices continue to diminish gradually, the traditional convective cooling with fluids would not be sufficient. Oftentimes, the sizes of the cooling systems needed are much bigger than the sizes of the electronic devices (Ghodoosi and Egrican [2]). Under these paradoxical circumstances, it has been demonstrated by Bejan and Lorente [3] that for small scales, the utilization of solid-body conduction with high thermal conductivity inserts is more effective than the best forced convection schemes. Constructal Theory concentrates on the geometric optimization of volume to point or point to volume flows encountered in engineering and science. The flow may be heat, fluid, goods, people etc. Constructal Theory introduces a hierarchical geometric flow

${ }^{*}$ Corresponding author

E-mail: campanto@yahoo.com

(C) 2016 International Association for Sharing Knowledge and Sustainability

DOI: 10.5383/ijtee.12.01.003 structure. Constructal Theory is established nowadays a mature field with a wide variety of applications to engineering, science and technology (Bejan and Lorente [4], Reis [5]). Conceptually, Constructal Theory has two relevant divisions. One is the use of the constructal law to predict and/or explain the occurrence of natural flow configurations, inanimate and animate. The other is the application of the constructal law as a physics principle to engineering applications. For the first time back in 1997, constructal law was invoked to minimize the thermal resistance between a volume and one point of an elementary rectangular heat-generating surface by means of high conductivity complete inserts connected at the center of a heat sink (Bejan [6,7]). Thereafter, designs with Constructal Theory were widely employed to optimize flow systems in engineering problems (see Bejan and Dan [8], Rocha [9]). As the heat power per unit volume or per unit surface in electronic devices increase, the heat dissipation becomes a crucial aspect. Thereby, the usage of a material with high thermal conductivity $k_{p}$ distributed in a base material with a relatively low thermal conductivity $k_{0}$ has been proposed in the sequence of References [10-23]. The conductive cooling with this methodology using high- $k$ channels in low- $k$ domains, has been largely investigated for different 
kinds of electronic devices. Many investigations have been carried out on rectangular [10-18], circular [9,19,20] and triangular domains [21] connected to solutions, configurations, conducting paths, cross-sections, etc. With regards to a cooling system for a rectangular domain, Ledezma et al. [10] postulated a solution by covering the heat generating volume with a series of building blocks, proceeding toward larger sizes (aka constructal approach). The authors inferred that a distribution with the shape of a tree for high-conductive material provides the optimum design. Dan and Bejan [11] minimized the time needed to discharge a volume to a sink in a constructal tree network by making appropriate changes in the geometry of flow path. Neagu and Bejan [12] reduced the global thermal resistance to flow between a volume and one point, by way of shaping the external boundary of each volume element, which resulted in a leaf-like tree structure with high-conductivity nerves and low-conductivity leaf material. Almogbel and Bejan [13] achieved significant improvement in connection to the global performance using a non-uniformly distribution of a high conductive material. An analysis based on the optimized variable cross-section of conducting paths of high- $k$ inserts was performed by Zhou et al. [14] showing that the heat resistance cannot always decrease when the complexity of the system increases. Wu et al. [15] obtained a more optimal construct to find the corresponding minimum heat resistance. Ghodoosi and Denton [16] devised an analytical solution to improve the accuracy of the heat transfer analysis in designing tree-like flow paths using the potent integral transform method. MathieuPotvin and Gosselin [17] proposed an evolutionary algorithm to optimize the heat resistance of a heat-generating area using high conductivity blades and later compared the results against those predicted by constructal theory. Several similarities were displayed by these authors in term of performances and geometries. Wei et al. [18] developed a method based on discrete variable cross-section via conducting paths to access more thermal current flows into the high conducting path and thus minimize the thermal resistance. Rocha et al. [9] provided an optimal analytical solution for the heat resistance in a sectorshaped elemental volume with the smallest dimension with a single high conductivity blade embedded into it. The elements were assembled into disc-shaped constructs to optimize the global resistance subjected to global constraints. The authors first believed that high-conductivity inserts caused the heat to flow in two perpendicular directions; then later used results with a radial insert case to optimize the disc resistance with branch inserts. Silva et al. [20] minimized the global thermal resistance of a disc cooled with rectangular high-k inserts that extended inward from the periphery. The authors showed analytically and numerically that the thermal resistance can be minimized with respect to the fin aspect ratio. Ghodoossi and Egrican [21] used a constructal solution method for cooling electronic systems formed with triangular heat generating spaces. Neagu and Bejan [22] maximized the amount of heat generating material along with minimizing the heat resistance of three-dimensional tree constructs. At very small length scales, Gosselin and Bejan [23], remarked that the situations become different and the parameter would be dependent on the shape and dimensions of the system. This work dealt with the cooling a two-dimensional heat generating conducting volume with one heat sink, such that the smallest features of the internal structure are so small that the conventional description of conduction breaks down. The effective thermal conductivity exhibits the "size effect," and is governed by the smallest structural dimension, which is comparable with the mean free path of the energy carriers. The construction approach reveals an internal multiscale structure shaped as a tree, where the spaces between the smallest branches are ruled by nanoscale heat transfer.
The present investigation focuses on a more general case involving the cooling of a solid disc having uniform internal heat generation. In this work, a degree of freedom is added to the problem by using incomplete inserts with high thermal conductivity instead of complete inserts with high thermal conductivity in the two radial and the branching configurations. As will be seen in the forthcoming sections, the incomplete inserts are superior when dealing with a wide range of parameters and limiting constraints. Also, the unique configuration is a good alternative where some practical limitations prohibit using complete inserts in the disc.

\section{Problem Formulation}

Consider a solid disc having uniform heat generation as sketched in Fig. 1. To cool the solid disc, the idea is to attach a heat sink at its center. The thermal conductivity of the solid disc is $k_{0}$ and the heat sink temperature $T_{0}$ has the lowest temperature throughout the ensemble. To minimize the global thermal resistance in the solid disc, materials with a high thermal conductivity $k_{p}$ can be utilized. From physical grounds, it is speculated that the maximum temperatures $T_{\max }$ likely happens along the rim of the solid disc. To optimize the heat resistance of the solid disc, first, the radial configuration of the incomplete inserts through the $k_{0}$-medium is dealt with; and second, the branching configuration is analyzed.

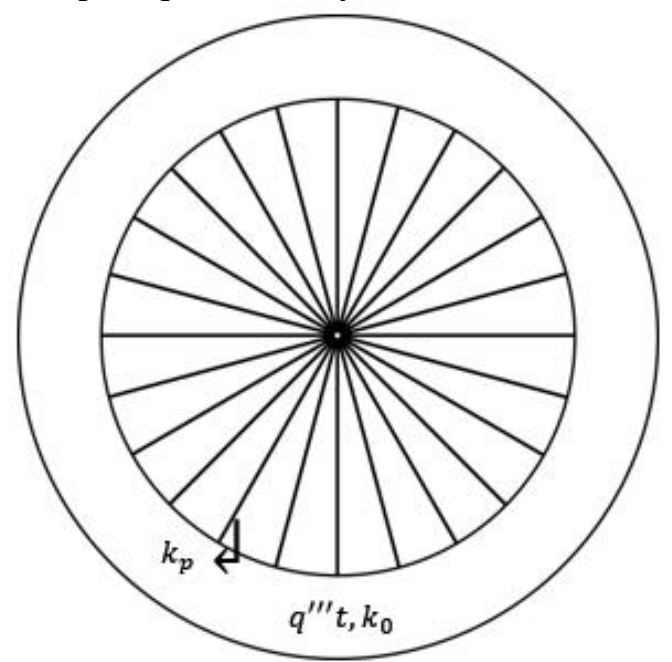

Figure 1a. Solid Disc with Uniform Heat Generation Connected to a Heat Sink at its Center

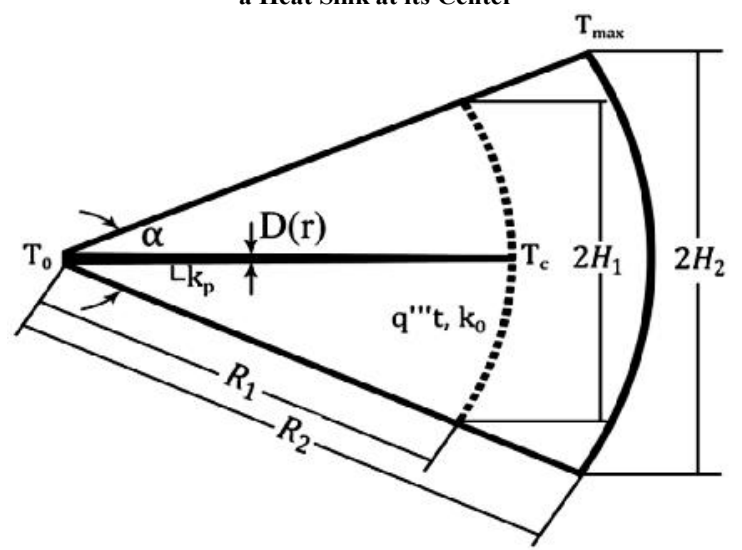

Figure 1b. Elemental Volume of a Circular Sector with a HighConductivity Material at its Center Line 


\subsection{Radial Pattern}

The concept of radial pattern is known as the simplest configuration in which materials with a high thermal conductivity $k_{p}$ are embedded as radial incomplete inserts through the solid disc with low thermal conductivity $k_{0}$ as shown on the left-side of Fig. 1. Exploiting the symmetry of the problem, the analysis commences with the construction of an elemental volume on the right-side in Fig. 1. The sector is surrounded by adiabatic radial sides (indicated as dashed lines) and the outer rim. The sector is approximated by means of an isosceles triangle with dimensions: base $2 \mathrm{H}_{2}$ and height $\mathrm{R}_{2}$. The analysis is separated into two parts.

\subsubsection{Region without High Thermal Conductivity Inserts}

For the portion of the element absent of inserts, the governing 2$\mathrm{D}$ heat conduction equation in cylindrical coordinates is

$$
\frac{\partial^{2} T}{\partial r^{2}}+\frac{1}{r} \frac{\partial T}{\partial r}+\frac{1}{r^{2}} \frac{\partial^{2} T}{\partial \theta^{2}}+\frac{q^{\prime \prime \prime}}{k_{0}}=0
$$

where $q$ "'stands for the heat generation per unit volume. Due to geometric and thermal symmetry, Eq. (1) is applied to half of the element where the proper boundary conditions are

$$
\begin{aligned}
& \left(\frac{1}{r} \frac{\partial T}{\partial \theta}\right)_{\left(r, \theta_{0}\right)}=0 \\
& \left(\frac{1}{r} \frac{\partial T}{\partial \theta}\right)_{(r, 0)}=0 \\
& \left(\frac{\partial T}{\partial r}\right)_{\left(R_{2}, \theta\right)}=0 \\
& T\left(R_{1}, \theta\right) \approx f(\theta) \approx f(y)
\end{aligned}
$$

In reference to the fourth boundary condition stated in Eq. (5), it implies a suitable approximation based on the fact that highly thermal conductive materials are responsible for the majority of heat flux and hence, the direction of heat flux is approximately perpendicular to the inserts. To demonstrate the consistency of the assumption, the analytical results will be compared against the numerical results in the forthcoming Section 5. Analogously, the governing $\mathrm{l} \cdot \mathrm{D}$ heat conduction in Cartesian coordinates

$\frac{1}{R_{1}^{2}} \frac{d^{2} T}{d \theta^{2}}+\frac{q^{\prime \prime \prime}}{k_{0}}=0$

provides a reasonable vehicle for the determination of the function $f(\theta)$ in Eq. (5). The, the proper boundary conditions for Eq. (6) are

$\frac{d T}{d \theta}=0$ at $\theta=\theta_{0}$

$T\left(R_{1}, 0\right)=T_{R 1}$

Integrating Fq. (6) subject to the boundary conditions in Eqs. (7) and (8), delivers the function $f(\theta)$ as

$T\left(R_{1}, \theta\right)=f(\theta)=T R_{1}+\frac{q^{\prime \prime \prime} R_{1}^{2}}{k_{0}}\left(\theta \theta_{0}-\frac{\theta^{2}}{2}\right)$
Owing that Eq. (1) is non-homogeneous, usage of the variable transformation (Arpaci [24])

$T(r, \theta)=\psi(r, \theta)+\varphi(r)$

is advisable.

The original 2-D heat conduction equation (l) with its nonhomogeneous term $\frac{q^{\prime \prime \prime}}{k_{0}}$ is conveniently separated into a homogeneous 2-D heat equation for the variable $\varphi$ :

$\frac{\partial^{2} \psi}{\partial r^{2}}+\frac{1}{r} \frac{\partial \psi}{\partial r}+\frac{1}{r^{2}} \frac{\partial^{2} \psi}{\partial \theta^{2}}=0$

and an inhomogeneous 1-D heat equation for the variable $\psi$ accounting for $\frac{q^{\prime \prime \prime}}{k_{0}}$,

$\frac{d^{2} \varphi}{d r^{2}}+\frac{1}{r} \frac{d \varphi}{d r}+\frac{q^{\prime \prime \prime}}{k_{0}}=0$

Accordingly, by virtue of Eq. (10), the boundary conditions in Eqs. (2)-(5) are replaced with

$\left(\frac{\partial \psi}{\partial \theta}\right)_{(r, 0)}=\left(\frac{\partial \psi}{\partial \theta}\right)_{\left(r, \theta_{0}\right)}=0$

$\psi\left(R_{1}, \theta\right)=f(\theta), \quad \varphi\left(R_{1}\right)=0$

$\left(\frac{\partial \psi}{\partial r}\right)_{\left(R_{2}, \theta\right)}=0, \quad\left(\frac{d \varphi}{d r}\right)_{\left(R_{2}\right)}=0$

First, double integrating Eq. (12) and applying the boundary conditions in Eqs. (14) and (15), the solution is

$\varphi(r)=\frac{-q \prime \prime \prime}{4 k_{0}} r^{2}+c_{1} \ln r+c_{2}$

where the constants of integration are

$c_{1}=\frac{q^{\prime \prime \prime} R_{2}^{2}}{2 k_{0}}, \quad c_{2}=\frac{q^{\prime \prime \prime} R_{1}^{2}}{4 k_{0}}-c_{1} \ln R_{1}$

Second, solving Laplace equation (11) by the method of separation of variables provides the 2-D temperature distribution

$\psi(r, \theta)=\frac{A_{0}}{2}+\sum_{n=1}^{\infty} A_{n}\left[+\left(\frac{R_{2}}{R_{1}}\right)^{2 \lambda_{n}}\left(\frac{r}{R_{1}}\right)^{-\lambda_{n}}\right] \cos \lambda_{n} \theta$

where the constants $A_{0}$ and $A_{n}$ are obtained from Fourier analysis. The expressions for $A_{0}$ and $A_{n}$ are

$$
\begin{aligned}
& A_{0}=2\left(T_{R_{1}}+\frac{1}{3} \frac{q^{\prime \prime \prime} R_{1}^{2} \theta_{0}^{2}}{k_{0}}\right)(19) \\
& A_{n}=\frac{2}{\left[1+\left(\frac{R_{2}}{R_{1}}\right)^{2 \lambda}\right]}\left\{\frac{T_{R 1} \sin n N \theta_{0}}{n N \theta_{0}}\right. \\
&+\frac{q^{\prime \prime \prime} R_{1}^{2}}{k_{0}}\left[\frac{\theta_{0} \sin n N \theta_{0}}{n N}\right. \\
&\left.+\frac{1}{(n N)^{2}}\left(\cos n N \theta_{0}-1\right)\right] \\
&-\frac{q^{\prime \prime \prime} R_{1}^{2}}{2 k_{0}}\left[\left(\frac{\theta_{0}}{n N}-\frac{2}{\theta_{0}(n N)^{3}}\right) \sin n N \theta_{0}\right. \\
&\left.\left.+\frac{2}{(n N)^{2}} \cos n N \theta_{0}\right]\right\}
\end{aligned}
$$


In here, the parameter $N=\frac{2 \pi R_{1}}{2 H_{1}}$ identifies the number of inserts or blades that are placed inside the solid disc with internal heat generation. Next, from Eq. (18), the temperature distribution at the location $r=R_{2}$ and $\theta=\theta_{0}$ becomes

$$
\begin{aligned}
T_{\max }-T_{R 1}=\frac{q^{\prime \prime \prime} R_{1}^{2} \theta_{0}^{2}}{3} & k_{0} \\
& -\sum_{\substack{n=1 \\
q_{0}(n N)^{2}}}^{\infty} \frac{4 q^{\prime \prime \prime} R_{1}^{2}}{k^{\prime \prime \prime}}\left(R_{2}^{2}-R_{2}^{2}\right) \\
& -\frac{\left.\left(R_{1}\right)_{1}\right)^{2 \lambda_{n}}}{4 k_{0}}\left(R_{2}^{2}-\cos n \pi\right. \\
& +\frac{q^{\prime \prime \prime} R_{2}^{2}}{2} \frac{R_{2}}{k_{0}} \ln \frac{R_{1}}{R_{1}}
\end{aligned}
$$

Alternatively, introducing the thermal conductivity of the solid disc material $k_{o}$, Eq. (21) can be non-dimensionalized. This leads to

$$
\begin{aligned}
\tilde{T}_{1}=\frac{\mathrm{T}_{\max }-\mathrm{T}_{0}}{\mathrm{q}^{\prime \prime \prime} \mathrm{A}_{1} / \mathrm{k}_{0}}= & \frac{1}{3} \frac{H_{1}}{R_{1}}-\sum_{n=1}^{\infty} \frac{4(-1)^{n}}{(n \pi)^{2}}\left[\frac{\left(R_{2} / R_{1}\right)^{\lambda_{n}}}{1+\left(R_{2} / R_{1}\right)^{2 \lambda_{n}}}\right] \frac{H_{1}}{R_{1}} \\
& -\frac{1}{4}\left[\left(\frac{R_{2}}{R_{1}}\right)^{2}-1\right] \frac{R_{1}}{H_{1}} \\
& +\frac{1}{2}\left(\frac{R_{2}}{R_{1}}\right)^{2} \ln \left(\frac{R_{2}}{R_{1}}\right) \frac{R_{1}}{H_{1}}
\end{aligned}
$$

where $A_{1}=H_{1} L_{1}$.

\subsubsection{Region with High Thermal Conductive Inserts}

In the core region, as the heat current flows toward the heat sink, its magnitude increase. For the element at the location $x=r$, the increment in $q$ is formulated by the energy balance

$-d q=h q^{\prime \prime \prime} t d r$

Here, $t$ is the thickness of the solid disc in the direction perpendicular to the plane in Fig. 1, and the product $h q^{\prime \prime \prime} t$ stands for the amount of heat gathered over the vertical surface $h t$, in which $h=\left(\frac{H_{1}}{R_{1}} r\right)$. The relation between the heat conducted and the local temperature gradient yields

$q=k_{p} \frac{D}{2} t \frac{d T}{d r}$

Due to the presence of incomplete inserts or blades, the boundary conditions are written as

$T=T_{0}$ at $r=0 \quad$ (25)

$\frac{q^{\prime \prime \prime} \pi\left(R_{2}^{2}-R_{1}^{2}\right) t}{2 N}=k_{p} \frac{D}{2} t \frac{d T}{d r}$ at $r=R_{1}$

Eliminating $q$ between Eqs. (23) and (24), integrating twice and using the pair of Eqs. (25) and (26), supplies the 1-D temperature distribution in the insert, $T(r)$,

$T(r)=\frac{-q^{\prime \prime \prime} r^{3}}{3 R_{1} k_{p} \phi_{1}}+\frac{q^{\prime \prime \prime} r}{k_{p} \phi_{1}}\left[\frac{\pi\left(R_{2}^{2}-R_{1}^{2}\right)}{H N}+R_{1}\right]+T_{0}$

Finally, due that $T=T_{R_{1}}$ at $r=R_{1}$, Eq. (27) reduces to

$T_{R 1}=\frac{-q^{\prime \prime \prime} R_{1}^{2}}{3 k_{p} \phi_{1}}+\frac{q^{\prime \prime \prime} R_{1}}{k_{p} \phi_{1}}\left[\frac{\pi\left(R_{2}^{2}-R_{1}^{2}\right)}{H N}+R_{1}\right]+T_{0}$
Because $\phi=\frac{N D R_{1}}{\pi R_{2}^{2}}$, the interconnection between $\phi_{1}$ and $\phi$ indicates that

$\phi_{1}=\phi\left(\frac{R_{2}}{R_{1}}\right)^{2}$

The heat resistance of the element in this part called $\tilde{T}_{2}$ is expressed as

$\tilde{T}_{2}=\frac{\mathrm{T}_{\max }-\mathrm{T}_{0}}{\mathrm{q}^{\prime \prime \prime} \mathrm{A}_{1} / \mathrm{k}_{0}}=\frac{1}{\bar{k} \phi}\left(\frac{R_{1}}{R_{2}}\right)^{2}\left(\frac{R_{1}}{H_{1}}\right)\left[\left(\frac{R_{2}}{R_{1}}\right)^{2}-\frac{1}{3}\right]$

Further, the total resistance of the elemental sector $\tilde{R}$ is obtained by adding Eqs. (22) and (30), eventually giving

$$
\begin{aligned}
\tilde{T}=\frac{1}{3} \frac{H_{1}}{R_{1}}-\sum_{n=1}^{\infty} \frac{4(-1)^{n}}{(n \pi)^{2}}\left[\frac{\left(R_{2} / R_{1}\right)^{\lambda_{n}}}{1+\left(R_{2} / R_{1}\right)^{2 \lambda_{n}}}\right]-\frac{1}{4}\left[\left(\frac{R_{2}}{R_{1}}\right)^{2}-1\right] \frac{R_{1}}{H_{1}} \\
+\frac{1}{2}\left(\frac{R_{2}}{R_{1}}\right)^{2} \ln \left(\frac{R_{2}}{R_{1}}\right) \frac{R_{1}}{H_{1}} \\
+\frac{1}{\tilde{k} \phi}\left(\frac{R_{1}}{R_{2}}\right)^{2}\left(\frac{R_{1}}{H_{1}}\right)\left[\left(\frac{R_{2}}{R_{1}}\right)^{2}-\frac{1}{3}\right]
\end{aligned}
$$

Here, $\frac{H_{1}}{R_{1}}$ indicates the aspect ratio and $\frac{R_{2}}{R_{1}}$ refers to the radius ratio. In the limit, when $\frac{R_{1}}{R_{2}}$ approaches unity, Eq. (31) provides the heat resistance of a solid disc with complete inserts. That is,

$$
\begin{array}{r}
\tilde{T}=\left[\frac{1}{3}-2 \sum_{n=1}^{\infty} \frac{(-1)^{n}}{(n \pi)^{2}}\right]\left(\frac{H_{1}}{R_{1}}\right)+\frac{2}{3 \tilde{k} \phi}\left(\frac{R_{1}}{H_{1}}\right) \\
=\frac{1}{2}\left(\frac{H_{1}}{R_{1}}\right)+\frac{2}{3 \tilde{k} \phi}\left(\frac{R_{1}}{H_{1}}\right)
\end{array}
$$

At this stage, it is convenient to view the heat conduction problem as an extremum problem. Then, the optimal heat resistance for a given value $\frac{R_{1}}{R_{2}}$ is determined by solving the equation $\frac{d \hat{T}}{d\left(\frac{H_{1}}{R_{1}}\right)}=0$. This step gives way to the following trascendental equation

$$
\begin{aligned}
& \frac{d \tilde{T}}{d\left(\frac{H_{1}}{R_{1}}\right)} \\
& =\left(\frac{1}{3}\right) \\
& +\sum_{n=1}^{\infty} \frac{4(-1)^{n+1}\left[\left(\frac{R_{2}}{R_{1}}\right)^{\lambda_{n}}\left(1+\left(\frac{R_{2}}{R_{1}}\right)^{2 \lambda_{n}}\right)+\lambda_{n}\left(\frac{R_{2}}{R_{1}}\right)^{3 \lambda_{n}} \ln \left(\frac{R_{2}}{R_{1}}\right)\left(1-\left(\frac{R_{1}}{R_{2}}\right)^{2 \lambda_{n}}\right)\right]}{(n \pi)^{2}\left(1+\left(\frac{R_{2}}{R_{1}}\right)^{2 \lambda_{n}}\right)^{2}} \\
& +\left(\frac{R_{1}}{H_{1}}\right)^{2}\left[\left(\frac{R_{2}}{R_{1}}\right)^{2}\left(\frac{1}{4}+\ln \left(\frac{R_{1}}{R_{2}}\right)^{1 / 2}\right)-\frac{1}{4}\right] \\
& \quad-\frac{1}{\tilde{k} \phi}\left(\frac{R_{1}}{R_{2}}\right)^{2}\left(\frac{R_{1}}{H_{1}}\right)^{2}\left[\left(\frac{R_{2}}{R_{1}}\right)^{2}-\frac{1}{3}\right] \\
& \quad=0
\end{aligned}
$$

which obviosly is of intricate form.

\subsection{Branching Pattern}

It is widely known that tree-shaped flows offer low resistance to deliver flows between one point and an area or between one point and a volume. In this sub-section, a different configuration involving materials with high thermal conduction is studied. Herein, the high conductivity material extends radially from the heat sink to the distance $L_{0}$ and then branches it out into a 
number of inserts " $\mathrm{n}$ " which cover the distance between $R_{1}$ and $L_{0}$ as observable in Fig. 2 . This particular structure has " $n$ " small sectors and a central core whose aspect ratios are $\frac{H_{1}}{L_{1}}$ and $\frac{H_{0}}{L_{0}}$, respectively. Moreover, there is a region in which no high conductive material resides, namely the ring. To compare the heat resistance of one branching case against the radial case, all assumptions made in sub-section 2.1 are held here as well. In our analysis, the region with inserts is divided into two parts: 1) a region with the branches and 2) the central part in which the stem is embedded.

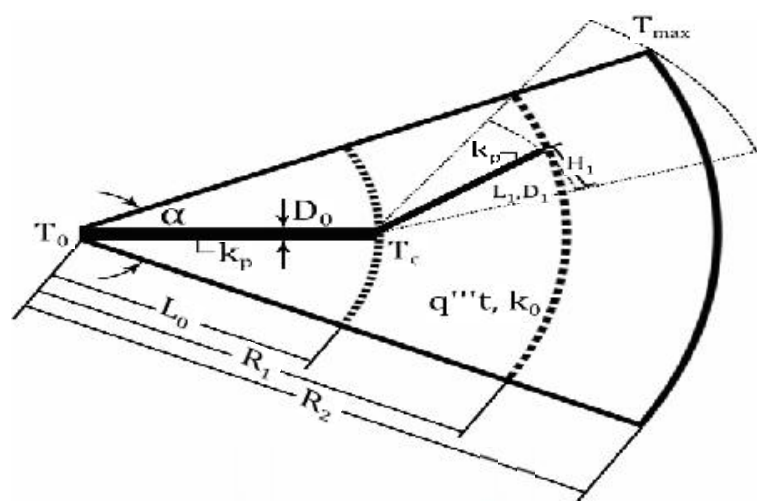

Figure 2. Elemental Surface of Branching Pattern, with $\mathbf{n}$ Branches $\left(L_{1}, D_{1}\right)$ and One $\operatorname{Stem}\left(L_{0}, D_{0}\right)$

2.2.1 Region with Branching Inserts

Assume that each sector with radius $\left(R_{2}-R_{1}+L_{1}\right)$ in which the branch is embedded, is slender enough. In light of this, the optimized aspect ratio of the radial pattern is applicable after the following replacements

$R_{1} \rightarrow L_{1}, \frac{D}{2} \rightarrow \frac{D_{1}}{2}, T_{0} \rightarrow T_{c}, R_{2} \rightarrow\left(R_{2}-R_{1}+L_{1}\right)$

are accounted for. Consequently, $\frac{H_{1}}{R_{1}}$ is replaced by $\frac{H_{1}}{L_{1}}$ and this step reveals that $\frac{H_{1}}{L_{1}}$ equals $\left(\frac{H_{1}}{R_{1}}\right)_{\text {opt }}$ as calculated before in subsection 2.1. In addition, it is worth defining $\phi_{1}=\frac{D_{1}}{H_{1}}$ and $A_{1}=$ $H_{1} L_{1}$ for mere convenience. Therefore, the total heat resistance of the region comprising the branching and the ring is similar to Eq. (31). That is,

$\tilde{T}_{1}=\frac{\mathrm{T}_{\max }-\mathrm{T}_{\mathrm{c}}}{\mathrm{q}^{\prime \prime \prime} \mathrm{A}_{1} / \mathrm{k}_{0}}=\frac{1}{3} \frac{H_{1}}{L_{1}}-\sum_{n=1}^{\infty} \frac{4(-1)^{n}}{(n \pi)^{2}}\left[\frac{\left(M / L_{1}\right)^{\lambda_{n}}}{1+\left(M / L_{1}\right)^{2 \lambda_{n}}}\right] \frac{H_{1}}{L_{1}}$ $-\frac{1}{4}\left[\left(\frac{M}{L_{1}}\right)^{2}-1\right] \frac{L_{1}}{H_{1}}$

$+\frac{1}{2}\left(\frac{M}{L_{1}}\right)^{2} \frac{L_{1}}{H_{1}} \ln \left(\frac{M}{L_{1}}\right)$

$+\frac{1}{\tilde{k}} \frac{\phi_{1}}{H_{1}}\left(\frac{L_{1}}{H_{1}}\right)\left[\left(\frac{M}{L_{1}}\right)^{2}\right.$

$\left.-\frac{1}{3}\right]$

where $M \equiv R_{2}-R_{1}+L_{1}$. Besides, the ratio $\left(\frac{M}{L_{1}}\right)$ is computed from the equation

$$
\begin{aligned}
\left(\frac{M}{L_{1}}\right)=1+\left(\frac{R_{2}}{L_{1}}\right)- & \left(\frac{R_{1}}{L_{1}}\right) \\
& =1 \\
& +\tilde{R}_{1}\left(\frac{H_{1}}{L_{1}}\right)_{o p t}^{\frac{1}{2}}\left[\left(\frac{R_{2}}{R_{1}}\right)\right. \\
& -1]
\end{aligned}
$$

\subsubsection{Core Region}

Since the boundary conditions for the central sector are different, it is not possible in this sub-section to use the $\left(\frac{H_{1}}{L_{1}}\right)_{\text {opt }}$ ratio obtained from sub-section 2.1. In this region, the approximation holds

$\left(\frac{H_{0}}{L_{0}}\right) \cong\left(\frac{\alpha}{2}\right)$,

$A_{0}=H_{0} L_{0}$

where $\alpha$ is the tip angle of the sector as illustrated in Fig. 2. This particular angle $\alpha$ turns out to be a function of other geometric parameters, as seeing in the equalities

$\alpha=\frac{2 \pi n}{N}=\frac{2 \pi n}{\left(\frac{2 \pi R_{1}}{2 H_{1}}\right)}=\frac{2 n}{\tilde{R}_{1}}\left(\frac{H_{1}}{L_{1}}\right)_{o p t}^{1 / 2}$

Back to Eq. (37), $A_{0}$ is the area of the central sector, which is calculated by the approximate expression

$A_{0} \cong n \hat{R}_{1}\left(\frac{H_{1}}{L_{1}}\right)_{o p t}^{1 / 2} A_{1}\left[1-\frac{1}{\tilde{R}_{1}}\left(\frac{H_{1}}{L_{1}}\right)_{o p t}^{-1 / 2}\right]^{2}$

where $\tilde{R}_{1} \equiv \frac{R_{1}}{A_{1}^{1 / 2}}$

Turning the attention to the central sector, the difference between the temperatures $T_{c}$ and $T_{0}$ is determined by way of the pair of equations Eqs. (23) and (24) found in sub-section 2.1.2. However, it is worth adding that the boundary condition at $r=$ $L_{0}$ is different. In equation form, the boundary condition is written as

$k_{p} D_{0} t\left(\frac{d T}{d r}\right)_{r=L_{0}} \cong q^{\prime \prime \prime} t\left[n A_{1}+\left(\frac{\alpha}{2}\right)\left(R_{2}^{2}-R_{1}^{2}\right)\right]$

Essentially, Eq. (40) implies that the total heat generated in the branching and outer ring regions is conducted to the heat sink using the radial high conductivity inserts in the core region. Finally, based on this argument, the heat resistance of the central sector is expressed as

$\tilde{T}_{2}=\frac{T_{c}-T_{0}}{q^{\prime \prime \prime} A_{1} / k_{0}}=\frac{\left[\tilde{R}_{1}-\left(\frac{L_{1}}{H_{1}}\right)_{o p t}^{1 / 2}\right]}{\tilde{k} \phi_{1} \widetilde{D}}\left(\frac{L_{1}}{H_{1}}\right)_{o p t}^{1 / 2} \times$
$\times\left\{\frac{2}{3} n \tilde{R}_{1}\left(\frac{H_{1}}{L_{1}}\right)_{o p t}^{1 / 2}\left[1-\frac{\left(\frac{L_{1}}{H_{1}}\right)_{o p t}^{1 / 2}}{\widetilde{R}_{1}}\right]^{2}+n \tilde{R}_{1}\left(\frac{H_{1}}{L_{1}}\right)_{o p t}^{1 / 2} \times\left[\left(\frac{R_{2}}{R_{1}}\right)^{2}-\right.\right.$
$1]\}$

where $\tilde{k}$ is the ratio of the thermal conductivities of the inserts to the solid disc and $\widetilde{D}=\frac{D_{0}}{D_{1}}$. Clearly, the heat resistance of the 
total element in Fig. 2 is simply the summation of Eqs. (35) and (41). Additionally, the relation between $\phi_{1}$ and $\phi$ is given by

$$
\begin{aligned}
\phi=\frac{N D_{1} L_{1}+\left(\frac{N}{n}\right)}{\pi R_{2}^{2}} & D_{0} L_{0} \\
& =\left(\frac{R_{1}}{R_{2}}\right)^{2} \frac{\phi_{1}}{\widetilde{R}_{1}}\left\{\left(\frac{L_{1}}{H_{1}}\right)_{o p t}^{1 / 2}\right. \\
& \left.+\frac{\widetilde{D}}{n}\left[\tilde{R}_{1}-\left(\frac{L_{1}}{H_{1}}\right)_{o p t}^{1 / 2}\right]\right\}
\end{aligned}
$$

\section{Discussion of Results}

In this section, all equations developed in Section 2 are used to obtain the heat resistance of a solid disc with radial and tributal configurations made with a high thermal conductivity material. In this context, the resistances are examined in order to check for extrema. Next, the optimal conditions associated with incomplete inserts are compared with those connected to complete inserts. This is done to reveal the importance of the former.

\subsection{Radial Pattern}

We used Eq. 31 to plot the variation of the thermal resistance with the aspect ratio $\frac{H_{1}}{R_{1}}$ for different values of $\frac{R_{1}}{R_{2}}$ in Fig. 3. The descending and ascending patterns of the curves indicate that there is a minimum for $\frac{R_{1}}{R_{2}}$ and more importantly the heat resistance increases as $\frac{R_{1}}{R_{2}}$ decreases. The level of complexity manifested in Eqs. (35) and (41) makes it difficult to find analytical solutions for the quantification of optimal aspect ratios and heat resistances. As a consequence, the optimal parameters are obtained numerically and are shown in the pair of Figs. 4 and 5. Moreover, by way of a curve fitting, the trio of Eqs. $(43,44,45)$ is obtained to facilitate the determination of the optimal conditions. From the pair of Figs. 4 and 5, it is observed that the optimum aspect ratios and the heat resistances decrease whenever $\frac{R_{1}}{R_{2}}$ and $\tilde{k} \phi$ increase. Specifically, when $\frac{R_{1}}{R_{2}}=1$, the optimal value of the aspect ratio as well as the heat resistance equals to $\frac{2}{(3 \bar{k} \phi)^{1 / 2}}$. The outcome of the curve fitting brings forth the following set of three correlation equations

$\left(\frac{H_{1}}{R_{1}}\right)_{\text {opt }}=0.540\left(\frac{R_{1}}{R_{2}}\right)^{-1.399}, \tilde{T}_{\text {opt }}=0.500\left(\frac{R_{1}}{R_{2}}\right)^{-1.435}$, for $\tilde{k} \phi=3$

$\left(\frac{H_{1}}{R_{1}}\right)_{o p t}=0.456\left(\frac{R_{1}}{R_{2}}\right)^{-1.482}, \tilde{T}_{o p t}=0.414\left(\frac{R_{1}}{R_{2}}\right)^{-1.528}$, for $\tilde{k} \phi=10$

$\left(\frac{H_{1}}{R_{1}}\right)_{\text {opt }}=0.417\left(\frac{R_{1}}{R_{2}}\right)^{-1.527}, \tilde{T}_{\text {opt }}=0.374\left(\frac{R_{1}}{R_{2}}\right)^{-1.578}$, for $\tilde{k} \phi=100$

sharing correlation coefficients R close to one.

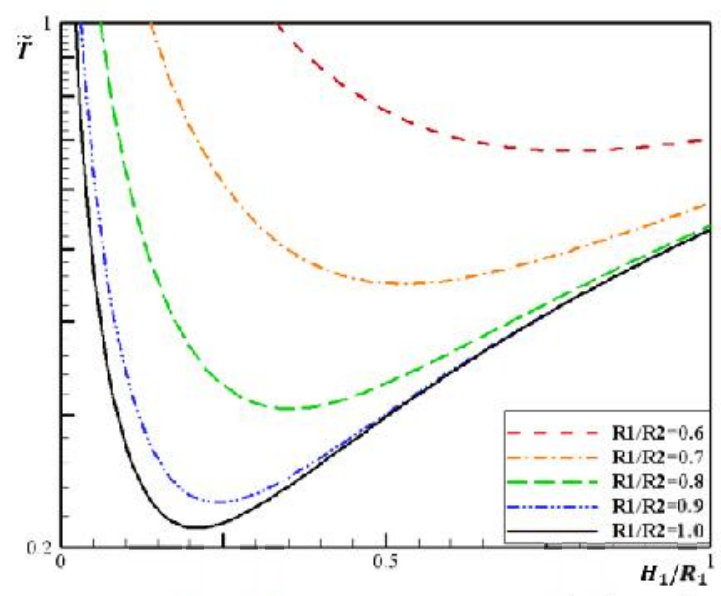

Figure 3. Variation of heat resistance with aspect ratio for $\widetilde{\boldsymbol{k}}=$ $300, \phi=0.1$ and different values of $R_{1} / R_{2}$

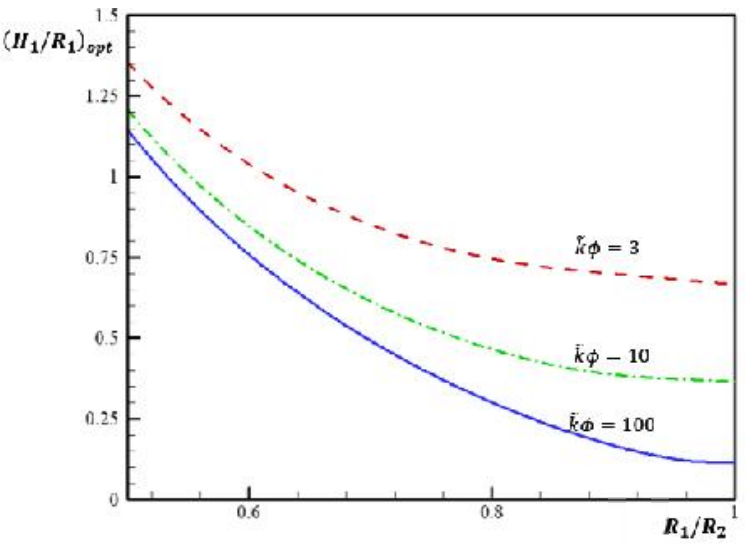

Figure 4. Optimal Aspect Ratio as a Function of $R_{1} / R_{2}$

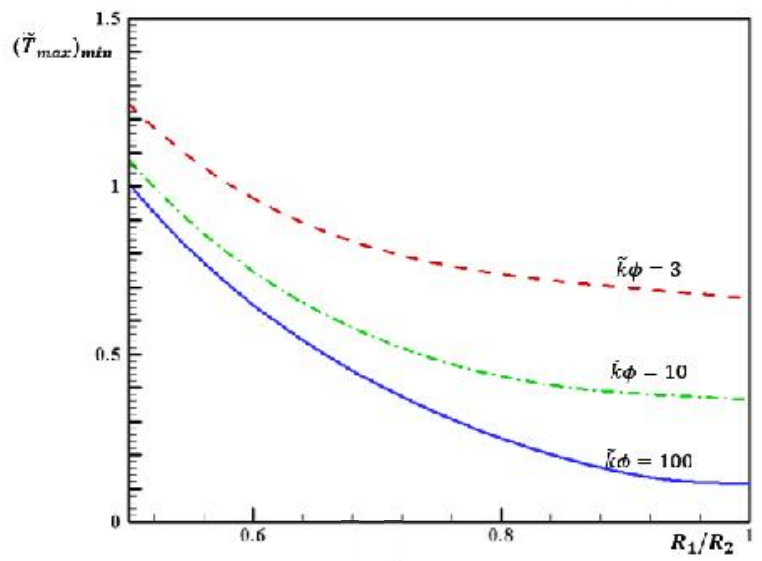

Figure 5. Variations of Optimal Heat Resistance with Respect to $R_{1} / R_{2}$

Figure 6 contains the optimal number of inserts, namely $N_{\text {opt }}=$ $\frac{\pi}{\left(\frac{H_{1}}{R_{1}}\right)_{o p t}}$. It is seen here that the optimal number of inserts $N_{\text {opt }}$ grows with increments in the two independent quantities $\tilde{k} \phi$ and $\frac{R_{1}}{R_{2}}$. When the area of the element $A_{s}$ is specified, the outer radius of the solid disc can be found from the relation 
$N A_{s}=\pi R_{2}^{2}$. Thereby, the optimal outer radius $\left(R_{2}\right)_{o p t}$ is given by the expression

$\left(R_{2}\right)_{\text {opt }}=\left[\left(\frac{R_{1}}{H_{1}}\right)_{o p t} A_{s}\right]^{1 / 2}$

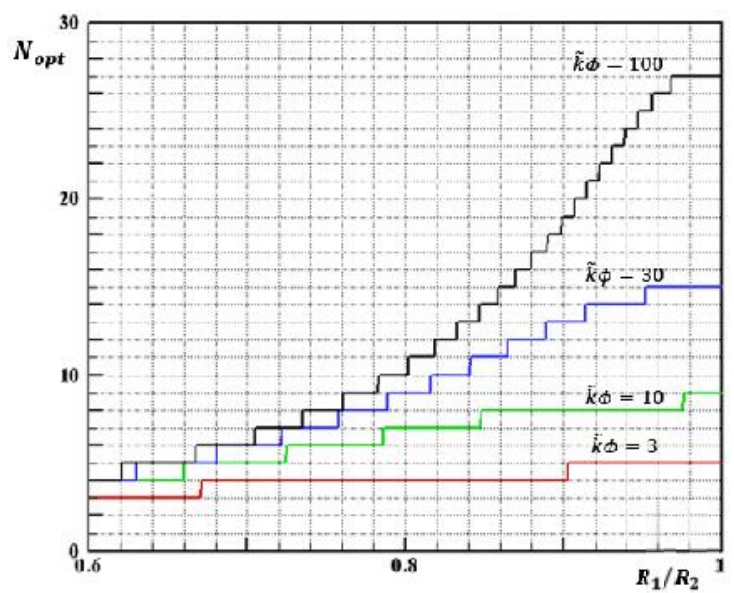

Figure 6. Optimal Number of Inserts as a Function of $R_{1} / R_{2}$

Fig. 7 displays this optimum radius $\left(R_{2}\right)_{\text {opt }}$ for a particular case specified by $A_{s}=0.1$. The reader should notice that the observable trend is similar to the one connected to an optimal number of inserts $N_{\text {opt }}$.

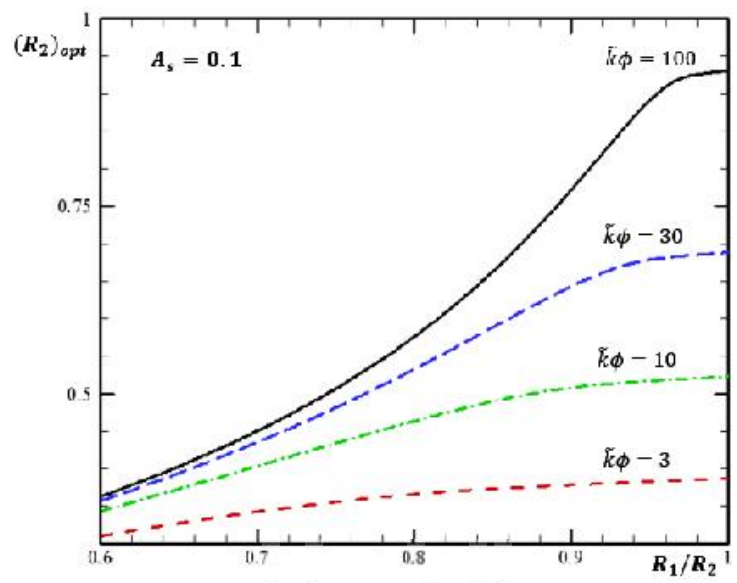

Figure 7. Optimal Radius of the Solid Disc as a Function of

$$
R_{1} / R_{2} \text { when } A_{s}=0.1
$$

Turning now the attention to the global heat resistance for radial pattern, $\tilde{T}_{G R, o p t}$, this quantity can be found from the relation

$\tilde{T}_{G R, o p t}=\frac{T_{\max }-T_{0}}{q^{\prime \prime \prime} \pi R_{2}^{2} / k_{0}}=\left(\frac{\tilde{T}}{\pi}\right)\left(\frac{R_{1}}{R_{2}}\right)^{2}\left(\frac{H_{1}}{R_{1}}\right)_{o p t}$

As seen in Fig. 8, the global heat resistance has an optimum point for each $\tilde{k} \phi$, which occurs at higher values of $\frac{R_{1}}{R_{2}}$ as $\tilde{k} \phi$ is elevated. A comparison between the complete and incomplete inserts heat resistances is depicted in Fig. 9. The performance of the incomplete insert heat resistance relative to the complete insert heat resistance is named $\mathrm{P}$ for short. The ensuing criterion is postulated as follows

$\mathrm{P}=\left|\frac{\text { incomplete insert heat resistance }- \text { complete insert heat resistance }}{\text { complete insert heat resistance }}\right| \times 100$

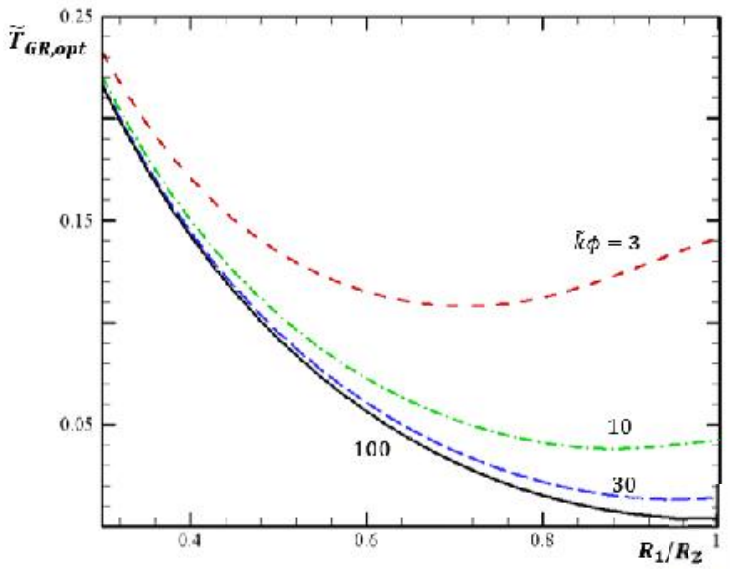

Figure 8. Optimal Global Heat Resistance as a Function of $\boldsymbol{R}_{1} / \boldsymbol{R}_{2}$

From the inspection of Fig. 9, it can be concluded that in each $\tilde{k} \phi$, there is a range of $\frac{R_{1}}{R_{2}}$ whose total heat resistance is smaller than the one belonging to a complete insert. Obviously, this is one of the benefits for using incomplete inserts; viz. in addition to placing the inserts in a smaller area, the heat performance of the system surpasses that of the heat performance of the complete insert.

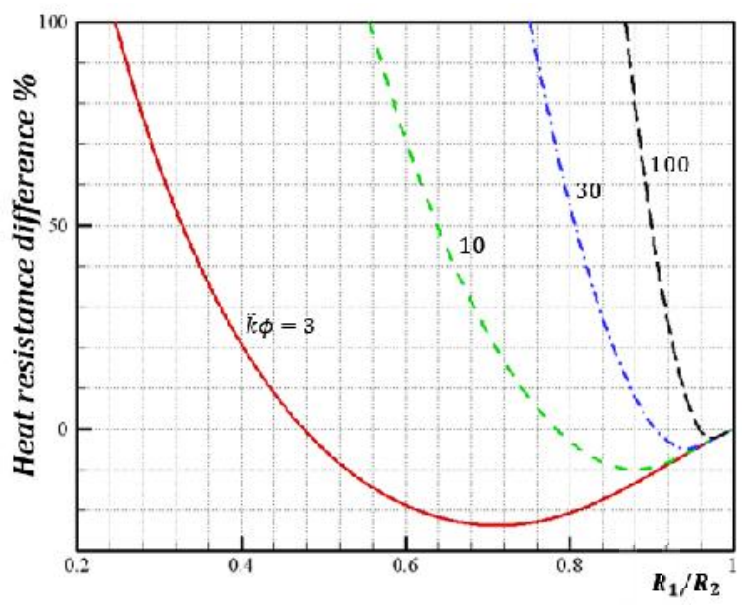

Figure 9. Difference between Global Heat Resistances with Complete and Incomplete Inserts as a Function of $R_{1} / R_{2}$

As illustrated in the tandem of Figs. 8 and 9, there is a minimum point in the optimum in the curve for total heat resistance, which happens at a specific value of $\tilde{k} \phi$. Fig. 10 elucidates the optimized values of the total heat resistance $\tilde{T}_{G R \text {,optopt }}$ and the corresponding values of $\left(\frac{R_{1}}{R_{2}}\right)_{\text {opt }}$. It is concluded that with increments in $\tilde{k} \phi$, the optimal total heat resistance shows a tendency to reduce. However, after exceeding the approximate 
value $\tilde{k} \phi=25$ (note that $\phi=0.1$ ), there is no significant decay in $\tilde{T}_{G R, o p t o p t}$.
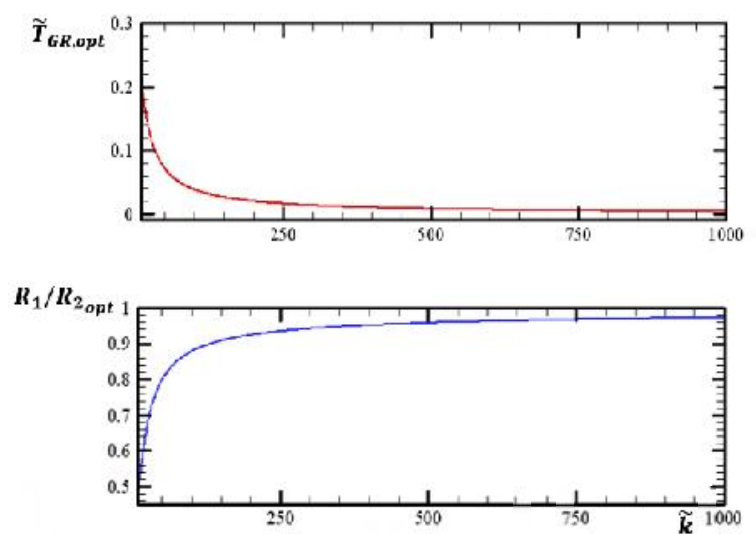

Figure 10. Optimized Values of Optimal Total Heat Resistance, $\widetilde{T}_{G h, o p t}$, and their Corresponding Values of $\left(R_{1} / R_{2}\right)_{o p t}$ when $\phi=$ 0.1

\subsection{Branching Pattern}

The combination of Eqs. 35 and 39 was instrumental in facilitating the plotting of the heat resistance for the branching configuration of high conductivity material. Shown in Fig. 11 is the heat resistance versus $\phi_{1}$ for fixed values of the parameters $\phi=0.1, \tilde{k}=300, n=2$ and $\tilde{R}_{1}=4$. From this figure, it is recognized that for each radius ratio $\frac{R_{1}}{R_{2}}$, there is a point at which the heat resistance passes through a minimum. In this context, the corresponding optimal values of $\phi_{1}$ and $\widetilde{D}$ are designated $\left(\phi_{1}\right)_{\text {opt }}$ and $(\widetilde{D})_{\text {opt }}$, respectively. Owing that the heat resistance in the branching configuration depends on the two geometrical parameters $\frac{R_{1}}{R_{2}}$ and $\tilde{R}_{1}$, the optimization process must be performed in association with these two parameters exclusively.

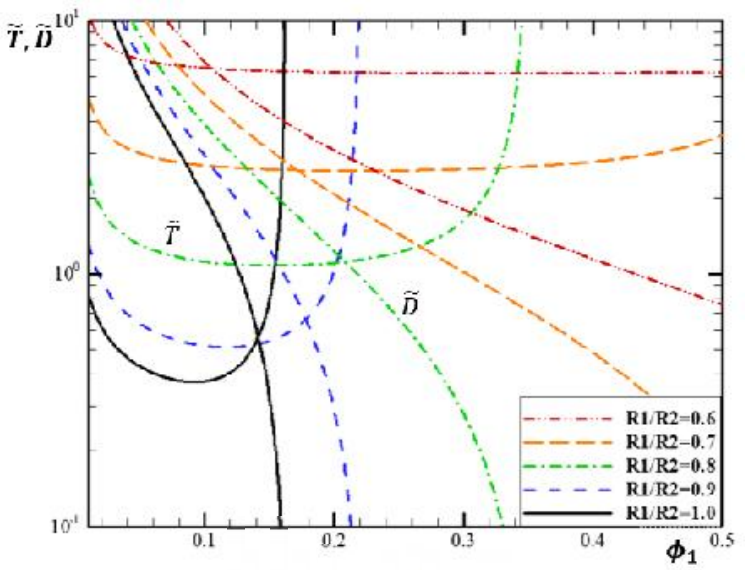

Figure 11. Heat Resistance and Insert Thickness Ratio for Different $\boldsymbol{R}_{1} / \boldsymbol{R}_{2}$ Values for a Combination of $n=2, \widetilde{R}_{1}=4, \widetilde{k} \phi=30$

3.2.1 Optimization of the Heat Resistance with Respect to the $\mathbf{R}_{1} / \mathbf{R}_{2}$ Ratio

In this sub-section, the heat resistance is optimized with respect to $\frac{R_{1}}{R_{2}}$. In this regard, Fig. 12 contains the optimum values of different parameters as a function of $\frac{R_{1}}{R_{2}}$. The optimal number of inserts $N_{\text {opt }}$ having length $L_{1}$ is $N_{o p t}=\pi \tilde{R}_{1}\left(\frac{L_{1}}{H_{1}}\right)_{o p t}^{1 / 2}$. It is seen in this figure that $N_{\text {opt }}$ augments with increments in $\frac{R_{1}}{R_{2}}$. The ratio of insert thicknesses found in Eq. 42 elevates as $\frac{R_{1}}{R_{2}}$ rises gradually. Further, when $\frac{R_{1}}{R_{2}}$ rises, this causes a decrement in the stem insert length, $L_{0, o p t}$. Further, the non-dimensional version of $L_{0, o p t}$ is obtained from the ratio

$\tilde{L}_{0, o p t}=\frac{L_{0, o p t}}{A_{1}^{1 / 2}}=\tilde{R}_{1}-\left(\frac{L_{1}}{H_{1}}\right)_{o p t}^{\frac{1}{2}}$

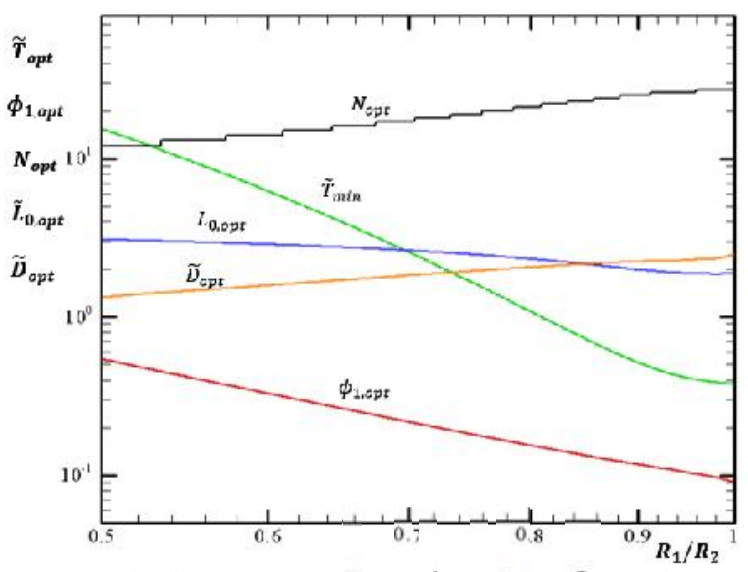

Figure 12. Optimum Parameters of Incomplete Inserts for a Combination of $\widetilde{R}=4, n=2, \widetilde{k} \phi=30$

The most important information that can be extracted from Fig. 12 is that the optimum heat resistance diminishes drastically as $\frac{R_{1}}{R_{2}}$ increases. Also, the aspect ratio of the area where the branches are allocated also diminish drastically as $\frac{R_{1}}{R_{2}}$ is increased. The global heat resistance for the branching patterns $\tilde{R}_{G B, o p t}$ can be evaluated using the expression

$\tilde{T}_{G B, o p t}=\frac{T_{\max }-T_{0}}{q^{\prime \prime \prime} \pi R_{2}^{2} / k_{0}}=\left(\frac{\tilde{T}}{\pi \tilde{R}_{1}^{2}}\right)\left(\frac{R_{1}}{R_{2}}\right)^{2}$

Displayed in Fig. 13 is the unfavorable effect posed by the incomplete inserts upon the global heat resistance. It is inferred that similar to the radial pattern, within a small range of $\frac{R_{1}}{R_{2}}$, the optimum global heat resistance of an incomplete structure is slightly less than that of the complete structure. Moreover, the optimum global heat resistance renders a minimum in a specific $\tilde{R}_{1}$. Hence, it is possible to achieve the optimal global heat resistance as viewed in Fig. 14. 


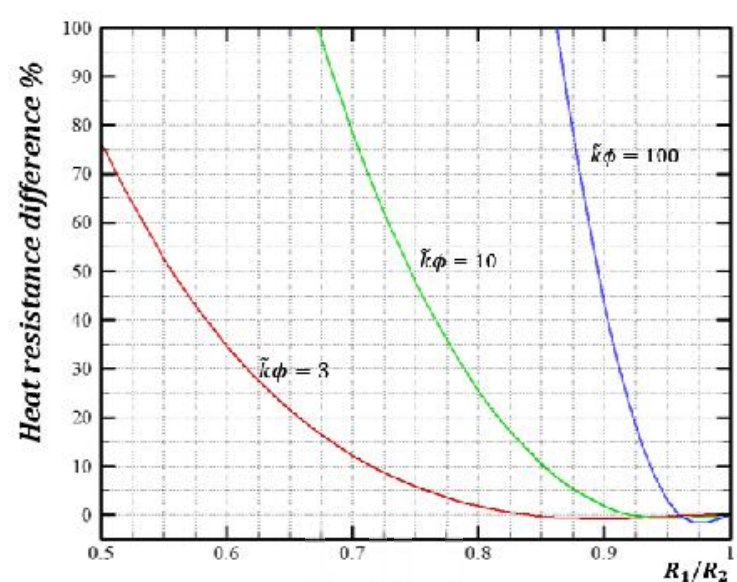

Figure 13. Optimal Total Heat Resistance Difference for Fixed $\widetilde{R}_{1}=4, n=2$

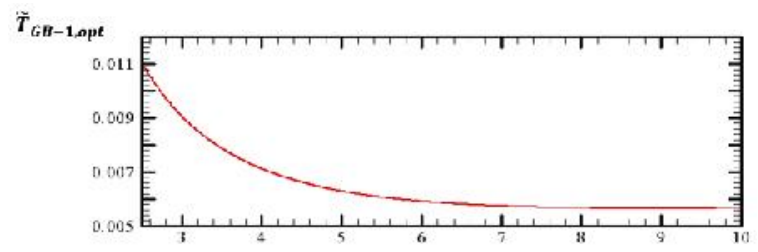

$\left(R_{1} / R_{2}\right)_{o p t}$

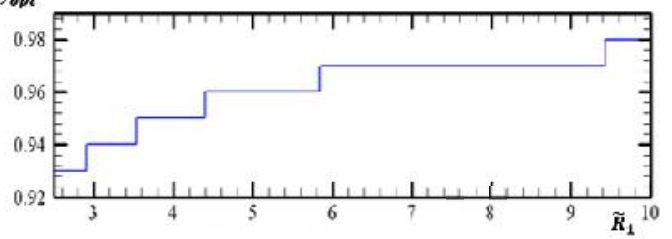

Figure 14. Optimized Values of Optimal Total Heat Resistance, $\widetilde{T}_{G E-1, o p t}$, and their Corresponding Values of $\left(R_{1} / R_{2}\right)_{o p t}$ for Fixed $\widetilde{k} \phi=30, n=2$

3.2.2 Optimization of the Heat Resistance with Respect to $\widetilde{\boldsymbol{R}}_{\mathbf{1}}$ Fig. 15 illustrates the optimal number of branches and the ratio of insert thicknesses. Scrutinizing this figure, it is evident that the optimal number of branches invigorates when the magnitude of $\tilde{R}_{1}$ grows. Moreover, it is evident that the trend exhibited by the $\widetilde{D}_{\text {opt }}$ variations depends on the numerical value of $\frac{R_{1}}{R_{2}}$. The variation of $\tilde{L}_{0, o p t}$ with $\tilde{R}_{1}$ for different values of $\frac{R_{1}}{R_{2}}$ is portrayed in Fig. 16. From this figure, we infer that upon reducing $\frac{R_{1}}{R_{2}}$ allows us to have cases with one level of bifurcation with lower $\tilde{R}_{1}$. The variations of $\phi_{1, o p t}$ and $\tilde{T}_{o p t}$ are plotted in Fig. 17 and the ensuing interpretation is that $\tilde{T}_{\text {opt }}$ is invigorated at higher values of $\tilde{R}_{1}$. However, $\phi_{1, \text { opt }}$ decreases slightly with $\tilde{R}_{1}$ because when $\phi_{1, o p t}$ is reduced, the amount of high thermal conductivity material allocated to the area $A_{1}$ diminishes. Hence, the optimum heat resistance is enhanced proportionately. These analyses were accomplished for other values of " $n$ " as well. From here, it may be concluded that $N_{\text {opt }}$ and $\tilde{L}_{0, o p t}$ are independent of " $n$ ".

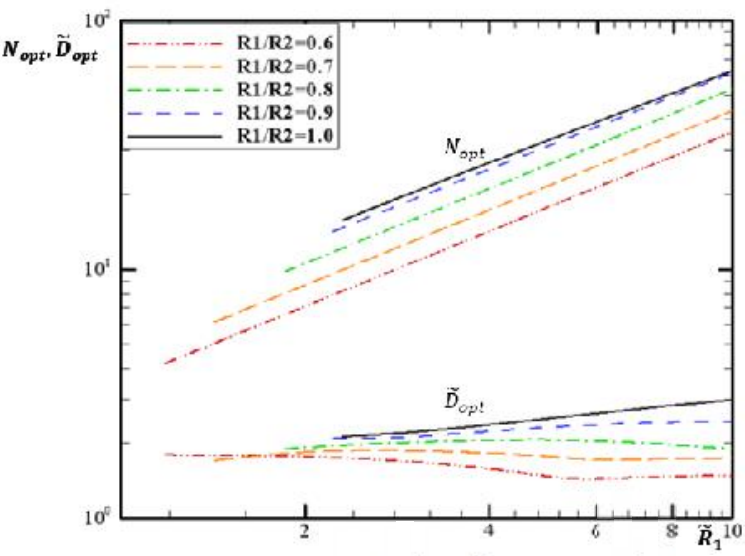

Figure 15. Optimal Number of Branches (Number of $A_{1}$ Elements) and the Insert Thickness Ratio at Different Values of $R_{1} / R_{2}$ for Fixed $n=2, \widetilde{k} \phi=30$

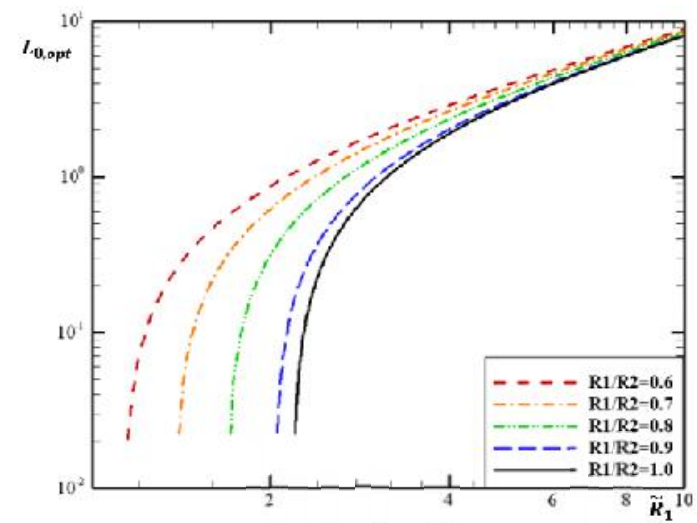

Figure 16. Variations of $L_{0, o p t}$ for Different Values of $R_{1} / R_{2}$ for Fixed $n=2, \tilde{k} \phi=30$

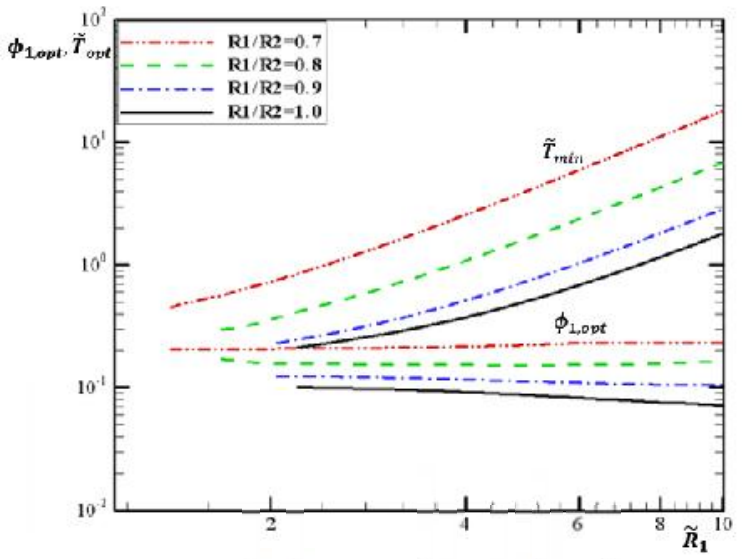

Figure 17. Minimal Heat Resistance, $\widetilde{T}_{m i t}$, and $\phi_{1 . o v t}$ for Different Values of $R_{1} / R_{2}$ and Fixed $n=2, \widetilde{k} \phi=30$

Fig. 18 embraces the variation of $\widetilde{D}_{\text {opt }}$ with $\tilde{R}_{1}$ for different values of " $n$ ". From analyzing this figure, it is palpable that those variations are merely insignificant. Moreover, the most interesting point is that despite the differences in $\widetilde{D}_{\text {opt }}$ for different values of " $n$ ", the values of $\frac{\widetilde{D}_{\text {opt }}}{n}$ are very close to each 
other and lie on the $\widetilde{D}_{\text {opt }}$ curve for " $\mathrm{n}$ " $=1$. Therefore, it may be concluded that the ratio $\frac{D_{0}}{D_{1}} \approx n$, holds true. This finding is relatively valid for all values of $\tilde{R}_{1}$ and signifies that the sum of $D_{1}$ values corresponds to a stem cross-section of diameter $D_{0}$. Shown in Fig. 19 is the optimal global heat resistance due to $\tilde{R}_{1}$ for radial (first point of each curve) and one level branching patterns at different values of $\frac{R_{1}}{R_{2}}$. The figure convincingly demonstrates that the global heat resistance for a tributary pattern is less accentuated than that of radial one. Also, the superiority of the complete blades related to the incomplete inserts for the most cases is self-evident in the figure. It can be emphasized that using incomplete inserts with $\frac{R_{1}}{R_{2}}=0.9$ whenever $\tilde{R}_{1}<3$ is advantageous. As this figure hints, each curve exhibits an optimum for each $\frac{R_{1}}{R_{2}}$. Therefore, it is possible to obtain the minimum for the optimized global heat resistance, as well as its corresponding value of $\tilde{R}_{1 o p t}$, as indicated in Fig. 20. In sum, it is clear that incrementing $\frac{R_{1}}{R_{2}}$ causes elevations in $\tilde{R}_{1 o p t}$ in conjunction with attenuations in $\tilde{T}_{G B-2, \text { optopt }}$.

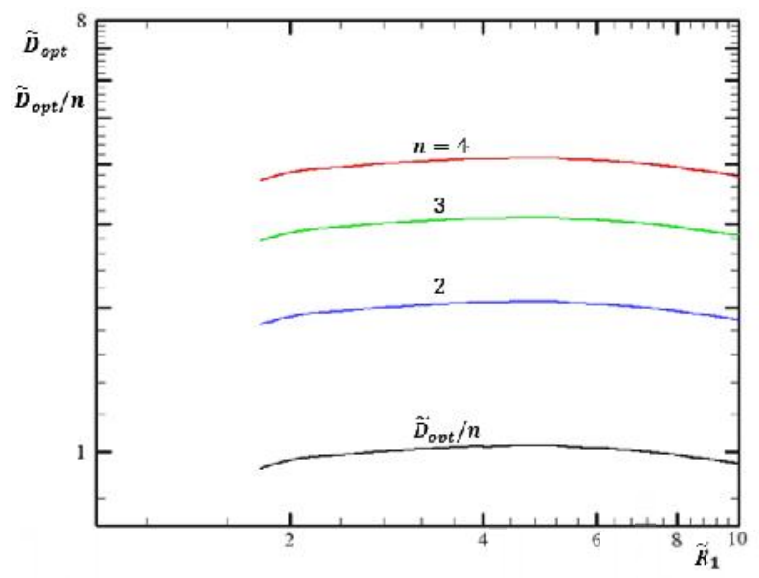

Figure 18. Optimal Values of Insert Thickness Ratios, $\widetilde{D}_{\text {opt }}$, and $\widetilde{D}_{\text {opt }} / \boldsymbol{n}$ at Different Values of $\mathrm{n}$ and Fixed $\widetilde{k} \phi=30, R_{1} / \boldsymbol{R}_{2}=0.8$.

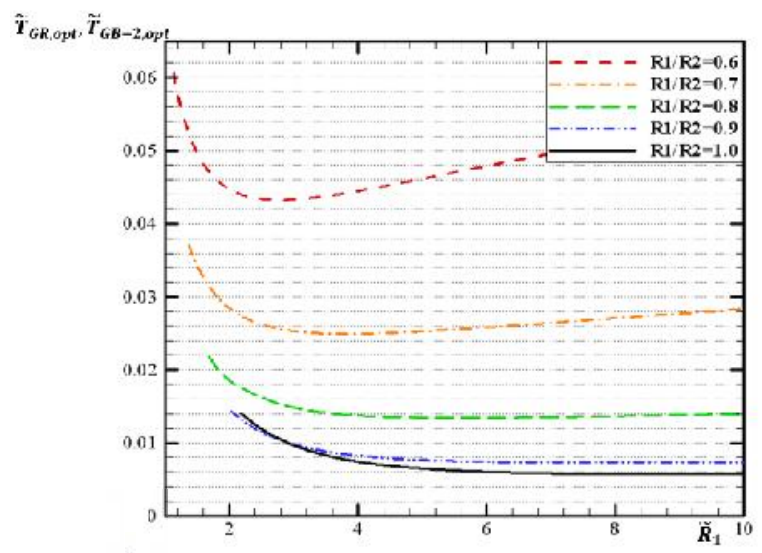

Figure 19. Optimal Total Heat Resistance at Different Values of $R_{1} / R_{2}$ for Radial and One Level Branching Cases
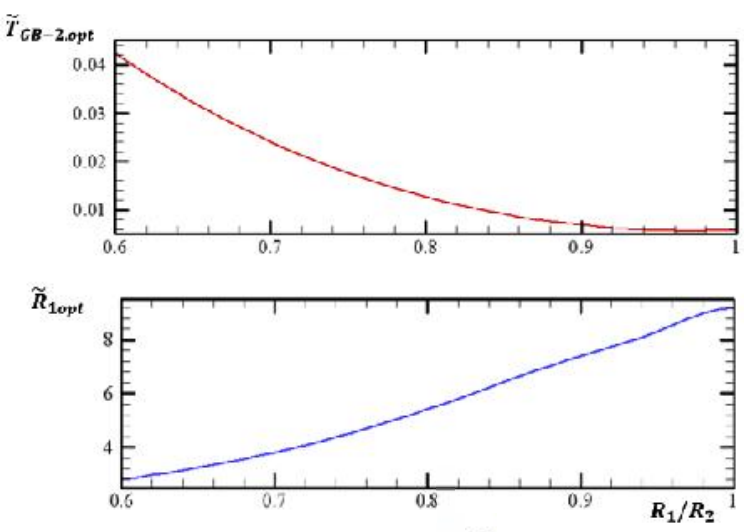

Figure 20. The Optimized Optimum Total Heat Resistance and the Corresponding $\widetilde{\boldsymbol{R}}_{1 \text { opt }}$

3.3 Numerical Results and Comparison

In this sub-section, the collection of numerical results are presented to validate/verify the analytical solutions. Herein, we are in the presence of a two-domain problem; first, the area with a material having low thermal conductivity $k_{0}$ and uniform heat generation $q^{\prime \prime \prime}$ and second, the inserts with high thermal conductivity $k_{p}$. Therefore, the heat conduction equations for the two-part ensemble are stated as follows

1) for the solid disc

$\nabla^{2} T+q^{\prime \prime \prime}=0$

2) for the inserts

$k_{p} \nabla^{2} T=0$

Exploiting symmetry, a sector of the solid disc is only considered for the numerical calculation with all boundaries modeled as adiabatic, namely $\frac{\partial T}{\partial n}=0$, where " $n$ " is the normal vector to each boundary. Since there is a heat sink placed at the center of the solid disc, the tip of the sector is maintained at a prescribed constant temperature, say $T_{\text {min }}$. Parallel to the analytical solution, the objective is to minimize the maximum temperature of the sector using geometric parameters, but using a numerical solution. Accordingly, the heat conduction equation for the sector was solved numerically using the finite element method (Zienkiewicz [25]) with the Partial Differential Equation Toolbox of the MATLAB code [26]. A computational mesh was constructed consisting of unstructured triangular elements. To identify the best mesh size, the mesh was refined sequentially until the mesh independent criterion

$\delta=\left|\frac{\tilde{T}_{\max }^{j+1}-\tilde{T}_{\max }^{j}}{\tilde{T}_{\max }^{j}}\right|<0.0005$

was satisfied. A typical grid independence test is summarized in detailed form in Table 1. In this context, the number of unstructured triangular elements was quadrupled in each step until the mesh independent criterion posed in Eq. (53) was satisfied. Using an appropriate number of triangular elements, the problem was solved numerically over a sector for cases with and without incomplete high conductivity inserts, as shown in the temperature contours in Fig. 21. The colors in the figure demonstrate clearly how the high conductive materials are capable of bringing down the mean and maximum temperatures of the solid disc. This behavior turns out to be quite interesting 
because of its beneficial applications in the cooling of electronic devices $[1,3]$.

Table 1 Grid Independence Test for a Typical Case characterized by $N=10, \widetilde{k}=100, L_{0}=0.5, \widetilde{D}=2.5, \phi=0.1$

\begin{tabular}{|c|c|c|}
\hline Number of Finite Elements & $\frac{r \alpha}{r m \alpha}$ & $\ddot{z}$ \\
\hline 1,092 & 0.128434 & 0.00267 \\
\hline 4,368 & 0.128777 & 0.00079 \\
\hline 17,472 & 0.128879 & 0.00026 \\
\hline 69,888 & 0.128913 & \\
\hline
\end{tabular}

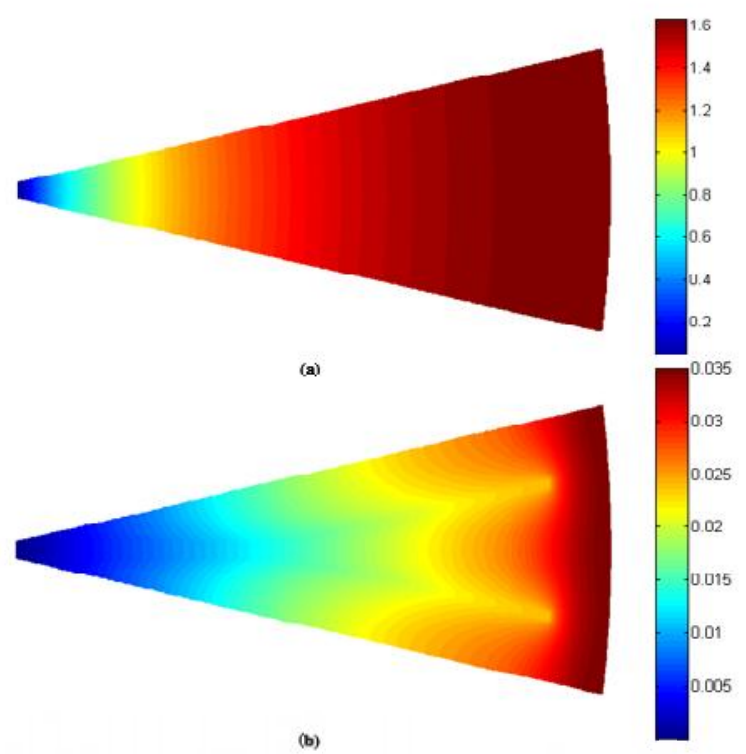

Figure 21. Temperature Contours for an Ensemble with $n=$ $2, \widetilde{D}=2.5, R_{1} / R_{2}=0.9, \widetilde{L}_{0}=2.5$ for (a) Disc without Inserts $\left(\widetilde{k}=1, \widetilde{T}_{m a r}=41.36\right)$ and (b) Dise with Inserts $\left(\widetilde{k}=300, \widetilde{T}_{m a}=\right.$ 0.89)

In order to validate the analytical exact results, the maximum temperature of the sector with a branching configuration of inserts was minimized with respect to the two geometric parameters $L_{0}$ and $\frac{D_{1}}{D_{2}}$. Essentially, this was done for two dissimilar cases characterized by $\tilde{k}=100$ and 300 articulated with fixed $\frac{R_{1}}{R_{2}}=0.8,0.9$ and 1 . The interrelated outcome is graphed in the Figs. 22 and 23 sequentially. To have a reasonable platform for comparison between the analytical results and the numerical results, the parameters were non-dimensionalized. Scrutinizing the two figures, a similar behavior is self-evident, which exemplifies that the heat resistance is susceptible to increments in $\tilde{R}_{1}$. In addition, an acceptable consistency pattern between the numerical and analytical results is observed and even more the consistency is more pronounced for situations characterized by $\frac{R_{1}}{R_{2}}=0.8$.

\section{Conclusions}

This study revolved around the analysis of steady conductive cooling of a solid disc with uniform heat generation by means of inserting incomplete highly conductive materials under the framework of the robust Constructal Theory. In the complex problem, the 1-D heat conduction equation cannot be applied directly. Instead, the 2-D conduction equation was solved analytically and the heat resistance was eventually optimized. It was categorically demonstrated that for a specific range of $\frac{R_{1}}{R_{2}}$ ratios, the optimal total heat resistance for incomplete inserts has a superior performance than those dealing with complete inserts located in the radial pattern. This matter constitutes a remarkable achievement, because when using less amount of high conductivity material, less total heat resistance is accessible. But this effect was a bit weaker for the branching case. In the radial case, as the optimal total heat resistance was an extremum at a specific value of $\frac{R_{1}}{R_{2}}$ and was later optimized. Moreover, all optimization estimates were performed for branching pattern inserts from two different standpoints. Finally, the maximum temperature of the sector was minimized numerically and afterward compared with the analytical results. In sum, acceptable levels of agreement were consistently observed for all situations examined.

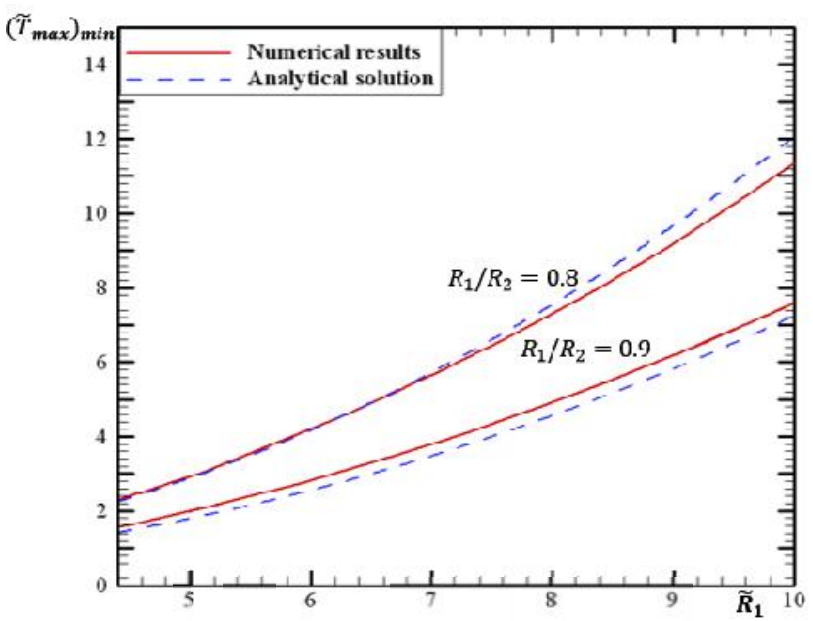

Figure 22. Comparison of Analytical and Numerical Results when $\widetilde{k}=100$ for two $\frac{R_{1}}{R_{2}}$ ratios $=0.8,0.9$

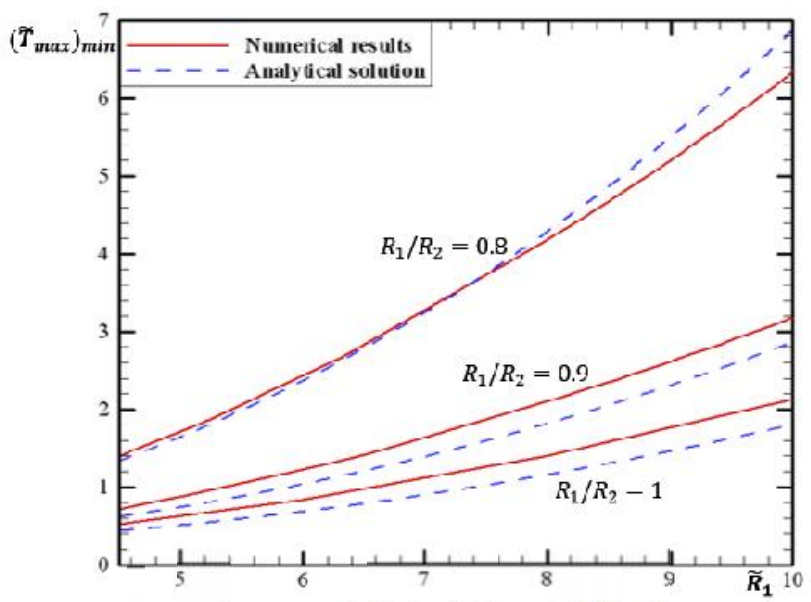

Figure 23. Comparison of Analytical and Numerical Results when $\widetilde{k}=300$ for three $\frac{R_{1}}{R_{2}}$ ratios $=0.8,0.9,1.0$ 


\section{References}

[1] Bar-Cohen, A. A. Watwe, R. S. Prasher, Heat Transfer in Electronic Equipment, Chapter 13, In Heat Transfer Handbook, eds. Bejan, A. D. Kraus, Wiley, New York, 2003.

[2] L. Ghodoosi, N. Egrican, Exact solution for cooling of electronics using constructal theory, Journal of Applied Physics 93 (8) (2003) 4922-4929.

[3] A.Bejan, S. Lorente, Design with Constructal Theory, Wiley, New York, 2008.

[4] Bejan, S. Lorente, Constructal theory of generation of configuration in nature and engineering, Journal of Applied Physics 100 (2006) Paper No. 041301.

[5] H. Reis, Constructal theory: From engineering to physics, and how systems flow develop shape and structure, Applied Mechanics Reviews 59 (5) (2006) Paper No. 269281.

[6] Bejan, Constructal-theory network of conducting paths for cooling a heat generating volume, International Journal of Heat and Mass Transfer 40 (4) (1997) 799-811.

[7] Bejan, Shape and Structure, from Engineering to Nature, pp. 52-61, Cambridge University Press, Cambridge, UK, 2000 .

[8] Bejan, N. Dan, Two constructal routes to minimal heat flow resistance via greater internal complexity, Journal of Heat Transfer 121 (1) (1999) 6-14.

[9] L. A. O. Rocha, S. Lorente, A. Bejan, Constructal design for cooling a disc-shaped area by conduction, International Journal of Heat and Mass Transfer 45 (8) (2002) 16431652 .

[10] G. A. Ledezma, A. Bejan, M. R. Errera, Constructal tree networks for heat transfer, Journal of Applied Physics 82 (1) (1997) 89-100

[11] N. Dan, A. Bejan, Constructal tree networks for the timedependent discharge of a finite-size volume to one point, Journal of Applied Physics 84 (6) (1998) 3042-3050.

[12] M. Neagu, A. Bejan, Constructal-theory tree networks of "constant" thermal resistance, Journal of Applied Physics 86 (2) (1999) 1136-1144.

[13] M. Almogbel, A. Bejan, Constructal optimization of nonuniformly distributed tree-shaped flow structures for conduction, International Journal of Heat and Mass Transfer 44 (22) (2001) 4185-4194.
[14] S. Zhou, L. Chen, F. Sun, Optimization of constructal volume-point conduction with variable cross section conducting path, Energy Conversion and Management 48 (1) (2007) 106-111.

[15] W. Wu, L. Chen, F. Sun, Heat-conduction optimization based on constructal theory, Applied Energy 84 (1) (2007) 39-47.

[16] L. Ghodoosi, J. C. Denton, Analytical solution for heat conduction problem in composite slabs and its implementation in constructal solutions for cooling of electronics, Energy Conversion and Management 48 (4) (2007) 1089-1105.

[17] F. Mathieu-Potvin, L. Gosselin, Optimal conduction pathways for cooling a heat-generating body: A comparison exercise, International Journal of Heat and Mass Transfer 50 (15-16) (2007) 2996-3006.

[18] S. Wei, L. Chen, F. Sun, The area-point constructal optimization for discrete variable cross-section conducting path, Applied Energy 86 (7-8) (2009) 1111-1118.

[19] L. A. O. Rocha, S. Lorente, A. Bejan, Conduction tree networks with loops for cooling a heat generating volume, International Journal of Heat and Mass Transfer 49 (15-16) (2006) 2626-2635.

[20] K. da Silva, C. Vasile, A. Bejan, Disc cooled with highconductivity inserts that extend inward from the perimeter, International Journal of Heat and Mass Transfer 47 (19-20) (2004) 4257-4263.

[21] L. Ghodoossi, N. Egrican, Conductive cooling of triangular shaped electronics using constructal theory, Energy Conversion and Management 45 (6) (2004) 811-828.

[22] M. Neagu, A. Bejan, Three-dimensional tree constructs of "constant" thermal resistance, Journal of Applied Physics 86 (12) (1999) 7107-7115.

[23] L. Gosselin, A. Bejan, Constructal heat trees at micro and nanoscales, Journal of Applied Physics 96 (10) (2004) $5852-5859$.

[24] V. S. Arpaci, Conduction Heat Transfer, Addison-Wesley, Reading, MA, 1966.

[25] O. C. Zienkiewicz, The Finite Element Method, McGrawHill Companies, New York, 1986.

[26] MATLAB-Partial Differential Equation Toolbox, The MathWorks, Inc., Natick, MA, 2002. 